\title{
IN PRAISE OF CONFIDENCE INTERVALS
}

\author{
David Romer
}

\section{ONLINE APPENDIX}

J anuary 2020

This appendix provides the details underlying the classification reported in Table 1 of the paper. It describes how the sample of papers was chosen, the categorization of specific papers, and complications and borderline cases.

As discussed in the main text, I consider equal numbers of papers published in the American Economic Review (AER), the J ournal of Political Economy (J PE), and the Quarterly J ournal of Economics (QJE) in 2019. Because the QJE publishes the fewest papers, I include all papers in the QJ E that meet my sample selection criteria; this yields 35 papers. To obtain the same number of papers from each of the other journals, I consider the first 35 papers published in the AER and in the J PE in 2019 that satisfy the sample selection criteria.

I exclude papers on three grounds. First, I drop comments, replies, errata, and special addresses (such as the American Economic Association presidential address and Nobel Prize lectures). Second, I exclude papers that do not perform any statistical estimation. Some papers in this group are entirely theoretical; some are empirical but purely descriptive; and some are a mix of the two. And third, I omit papers where I am thanked for comments, on the grounds that the presentation in these papers may have been affected by my views and so may not be representative of current "normal" practice in economics.

These rules lead me to drop to 45 papers: 35 papers that do not perform statistical estimation, 3 errata, 3 papers where I am thanked for comments, 2 replies, 1 comment, and 1 special address.

One issue that arises in classifying the papers concerns cases where papers mention regression estimates that the authors do not view as important enough to include in tables and 
figures, and so are reported in the text or in footnotes. In these cases, normal practice is to report both the point estimates and the standard errors. Because this provides no information about the standard errors or confidence intervals for papers' main estimates (and because it is almost inevitable when regression results are only reported in the text or footnotes), I do not count such cases as discussions of standard errors. Similarly, if a paper reports tests of the equality of coefficients but does not discuss the implications of its estimates for other parameter values besides zero, I do not classify the paper as discussing confidence intervals or standard errors.

More broadly, any categorization of papers into just six categories (the five shown in Table 1 plus the papers excluded from the sample) is inherently crude. I try to address this challenge in two ways. First, I rely on rules as much as possible. For example, I classify an empirical paper as descriptive (and therefore excluded from the sample) if it does not report any estimates together with standard errors or confidence intervals (or $\mathrm{p}$ values or t-statistics) in any tables or figures, even if some of its descriptive statistics can be thought of as parameter estimates. Second, when my rules do not lead to a clear categorization, I try to err on the side of putting a paper in a category corresponding to greater emphasis on standard errors. For example, suppose that at one point in a long paper, in the discussion of results presented in a table, the point estimate and standard error for a parameter that the paper does not emphasize is reported in the text, and that there are no other mentions of standard errors or confidence intervals. I classify such a paper as mentioning standard errors in passing even though they are virtually absent from the text. Similarly, if a paper reports but does not discuss the confidence interval for one of its main estimates in the conclusion but nowhere else, I classify the paper as discussing confidence intervals prominently.

Nonetheless, my classifications will not be perfectly "fair" to every paper. For example, a paper that is largely theoretical but that reports a few motivating regressions in a table in the introduction and does not mention the standard errors in the text will be categorized as not discussing standard errors or confidence intervals (a classification that is clearly technically 
correct, but not very informative).$^{1}$

The remainder of this appendix is a paper-by-paper description of whether papers are included in the sample and of my classification of the included papers into the different categories. If the paper is excluded, I explain the reason. If it is included and the classification is not completely straightforward, I explain the reasoning behind my decision and discuss any complications.

The purpose of choosing the papers I consider is to try to obtain a representative sample of leading recent empirical work, and not to evaluate specific papers in any way. Closely related, grouping papers into six categories does not reflect the nuances of each paper. I therefore identify papers just by journal, issue number, and paper order within the issue. For example, AER, issue 3, Paper 4 is the fourth paper in the third issue of the AER in 2019. Readers who want to check my classifications can use my paper identifiers to find the specific papers.

\section{American Economic Review}

\section{AER, Issue 1}

Paper 1

Classification: Discusses neither confidence intervals nor standard errors.

Comment: The paper includes some statements along the lines of, "zero effects lie outside of a 99 percent confidence interval" (p. 23). But this is just a way of saying that the p value is less than 0.01; it does not give the actual confidence interval.

\section{Paper 2}

Classification: Discusses neither confidence intervals nor standard errors.

Comment: In two places in the text (pp. 74 and 80) and in numerous places in the footnotes, the paper gives standard errors in reporting estimates from minor regressions that are not presented in tables or figures.

Paper 3

Classification: Excluded.

Comment: The paper is entirely theoretical.

1 Papers that never discuss standard errors or confidence intervals, but where the confidence intervals are so narrow that the economic interpretation of any point within them would be virtually the same, are classified as not mentioning point estimates or standard errors. I regard this classification as appropriate: by not discussing confidence intervals, the papers do not convey that there is very strong evidence against any economically interesting hypotheses about the parameter values other than ones that are very close to the point estimates. That is, in such cases not discussing confidence intervals potentially understates the importance of papers' findings. 
Paper 4

Classification: Discusses neither confidence intervals nor standard errors.

Paper 5

Classification: Discusses neither confidence intervals nor standard errors.

Comment: The paper is almost entirely theoretical. However, the introduction presents some statistical estimates of parameters. Thus the paper is marginal for inclusion in the sample. Because the table reporting the statistical estimates includes standard errors, the paper fits the criteria for inclusion in the sample. The text does not mention standard errors or confidence intervals associated with its statistical estimates.

Paper 6

Classification: Excluded.

Comment: The paper is entirely theoretical.

Paper 7

Classification: Discusses confidence intervals prominently.

Comment: One of the paper's central results is a negative one. It emphasizes not just that the point estimates are not statistically significantly different from zero, but also that the magnitude of the implied effects if the parameters are equal to the values at the upper and lower ends of the 95 percent confidence intervals are economically small. Thus the paper discusses confidence intervals for many of its main estimates.

Paper 8

Classification: Discusses confidence intervals in passing; does not discuss standard errors.

Comment: N. 31 (p. 306) reports the confidence interval for the difference between two parameters in one specification. The issue of whether the parameters are equal is of moderate importance in the paper.

Paper 9

Classification: Excluded.

Comment: The paper is almost entirely theoretical. The estimation of one regression is mentioned briefly, with no discussion of confidence intervals or standard errors (p. 300). Thus the paper is marginal for inclusion in the sample. Because the paper does not report standard errors or confidence intervals in any tables or figures, it is excluded from the sample.

Paper 10

Classification: Excluded.

Comment: The paper is a reply.

\section{AER, Issue 2}

Paper 1

Classification: Discusses standard errors prominently; does not discuss confidence intervals.

Comment: The paper reports standard errors for two important parameters in the text (p. 344). The parameter estimates and standard errors for those parameters are not reported in any tables or figures; thus one could reasonably say that the paper discusses standard errors in passing rather than prominently. The paper does not discuss confidence intervals.

Paper 2

Classification: Discusses standard errors prominently; does not discuss confidence intervals. 
Paper 3

Classification: Discusses neither confidence intervals nor standard errors.

Paper 4

Classification: Excluded.

Comment: I am thanked for comments.

Paper 5

Classification: Discusses neither confidence intervals nor standard errors.

Comment: The paper is largely theoretical, with quantitative applications to particular settings. Because the applications are based on parameters that are estimated statistically, the key estimates for the applications come with associated confidence intervals, which are reported in the paper's main tables (pp.509, 512, and 516). These confidence intervals (nor any other confidence intervals or standard errors) are not mentioned in the text.

Paper 6

Classification: Discusses neither confidence intervals nor standard errors.

Paper 7

Classification: Discusses confidence intervals prominently.

Comment: The paper is primarily theoretical, but performs a small amount of parameter estimation, with standard errors reported in two tables (pp. 573- 577). The confidence intervals for key estimates are reported in the text. The paper is marginal for inclusion in the sample.

Paper 8

Classification: Excluded.

Comment: The paper's data is entirely descriptive. Some of those descriptions can be thought of as parameter estimates (see Figures 13 and 14 on pp. 613- 614, for example). However, because the paper does not report standard errors or confidence intervals in its figures, I classify the paper as descriptive. The paper is marginal for inclusion in the sample. If it were included, it would be classified as discussing neither confidence intervals nor standard errors.

Paper 9

Classification: Discusses neither confidence intervals nor standard errors.

Paper 10

Classification: Excluded.

Comment: The paper is entirely theoretical.

Paper 11

Classification: Excluded.

Comment: The paper is entirely theoretical.

\section{AER, Issue 3}

Paper 1

Classification: Discusses neither confidence intervals nor standard errors.

Comment: In one footnote (n. 27, p. 766), the paper gives standard errors in reporting estimates from two minor regressions that are not presented in tables or figures.

Paper 2

Classification: Discusses standard errors prominently; does not discuss confidence intervals. Comment: The paper reports standard errors in numerous places, including in the 
introduction.

Paper 3

Classification: Discusses standard errors prominently; does not discuss confidence intervals.

Comment: The paper reports standard errors for two important parameters in the text (p. 828). (In addition, the paper reports two t-statistics in the introduction, but they are not reported with the associated point estimates. Thus they provide no information about magnitudes.)

Paper 4

Classification: Excluded.

Comment: The paper's data analysis is descriptive. Some of those descriptions can be thought of as parameter estimates (see Table 4 on p. 869, for example). However, because the paper does not report standard errors or confidence intervals in its tables, I classify the paper as descriptive. The paper is marginal for inclusion in the sample. If it were included, it would be classified as discussing neither confidence intervals nor standard errors.

Paper 5

Classification: Excluded.

Comment: The paper is entirely theoretical.

Paper 6

Classification: Excluded.

Comment: The paper is entirely theoretical.

Paper 7

Classification: Discusses neither confidence intervals nor standard errors.

Paper 8

Classification: Excluded.

Comment: The paper is entirely theoretical.

Paper 9

Classification: Discusses neither confidence intervals nor standard errors.

Paper 10

Classification: Discusses neither confidence intervals nor standard errors.

Paper 11

Classification: Excluded.

Comment: The paper is entirely theoretical.

Paper 12

Classification: Excluded.

Comment: The paper is a comment.

Paper 13

Classification: Excluded.

Comment: The paper is a reply. 


\section{AER, Issue 4}

Paper 1

Classification: Excluded.

Comment: The paper is the American Economic Association presidential address.

Paper 2

Classification: Excluded.

Comment: The paper is entirely theoretical.

Paper 3

Classification: Discusses neither confidence intervals nor standard errors.

Paper 4

Classification: Discusses confidence intervals prominently.

Comment: The paper discusses the upper bounds of the confidence intervals for some parameter estimates for which the point estimates are small (pp. 1291 and 1311).

Paper 5

Classification: Excluded.

Comment: The paper is entirely theoretical.

Paper 6

Classification: Excluded.

Comment: The paper is entirely theoretical.

Paper 7

Classification: Discusses neither confidence intervals nor standard errors.

Comment: Although the paper is mainly theoretical, with some descriptive data analysis, one part performs statistical estimation and includes two tables that report point estimates and standard errors (pp. 1417- 1420). This material does not mention confidence intervals or standard errors in the text. The paper is marginal for inclusion in the sample.

Paper 8

Classification: Discusses neither confidence intervals nor standard errors.

Paper 9

Classification: Discusses neither confidence intervals nor standard errors.

Comment: Although the paper is largely empirical, only a relatively small part estimates parameters and reports point estimates and standard errors in tables or figures (pp. 1469-1471). This material does not mention confidence intervals or standard errors in the text. The paper is marginal for inclusion in the sample.

Paper 10

Classification: Discusses neither confidence intervals nor standard errors.

Paper 11

Classification: Discusses standard errors in passing; does not discuss confidence intervals.

Comment: In one place (p. 1544), the paper reports the standard errors for three parameter estimates that are somewhat important to its results, but not central.

Paper 12

Classification: Discusses neither confidence intervals nor standard errors. 
Paper 13

Classification: Excluded.

Comment: The paper is an erratum.

\section{AER, Issue 5}

Paper 1

Classification: Discusses neither confidence intervals nor standard errors.

Paper 2

Classification: Discusses neither confidence intervals nor standard errors.

Comment: At one point, the paper refers to a "precisely estimated 0 coefficient" (p. 1671). However, since the upper bound of the confidence interval for the parameter almost reaches a value that the paper interprets as quantitatively large, it appears that "precisely estimated 0 " is being used to mean "highly insignificant." Nonetheless, the paper could arguably be classified as discussing confidence intervals in passing. In addition, in several places the paper reports standard errors from minor regressions that are not reported in any tables or figures (pp. 1671n, 1676n, and 1680n).

Paper 3

Classification: Discusses neither confidence intervals nor standard errors.

Comment: At one point, the paper reports standard errors from some minor regressions that are not reported in any tables or figures (p. 1698). At another point, it describes how the standard errors differ across two specifications, but without providing any quantitative information about the magnitude of the standard errors (p. 1704).

Paper 4

Classification: Discusses standard errors in passing; does not discuss confidence intervals.

Comment: The text of the paper reports the standard error of one regression coefficient (p. 1744).

Paper 5

Classification: Discusses neither confidence intervals nor standard errors.

Comment: In several places, the paper describes how changes affect the standard errors without providing information about their magnitude (pp. 1782, 1784n, and 1788n).

Paper 6

Classification: Discusses confidence intervals in passing; does not discuss standard errors.

Comment: At one point, the paper reports that the posterior distributions (effectively, confidence intervals) for some parameters for one set of estimates are similar to those from an earlier study (p. 1825). (Also, at another point, the paper states for the 90 percent interval for the estimate of a parameter is above zero for some period, but this is essentially just a way of saying that the estimate is significantly greater than zero; see p. 1832.)

Paper 7

Classification: Discusses neither confidence intervals nor standard errors.

Paper 8

Classification: Discusses confidence intervals prominently.

Comment: A central focus of the paper is on the uncertainty concerning certain key parameters. 


\section{J ournal of Political Economy}

\section{JPE, Issue 1}

Paper 1

Classification: Discusses neither confidence intervals nor standard errors.

Comment: Only one part of the paper reports parameter estimates with standard errors or confidence intervals in tables or figures (pp. 20-24). That section does not mention confidence intervals or standard errors in the text.

Paper 2

Classification: Discusses standard errors prominently; does not discuss confidence intervals.

Paper 3

Classification: Discusses neither confidence intervals nor standard errors.

Comment: At one point, in discussing some minor regressions that are only reported in an online appendix, the paper reports that "the standard errors are too large to rule [out] a substantial negative effect" (p. 128). Because this statement concerns regressions not reported in tables or figures in the main paper and there are no other references to confidence intervals or standard errors, I classify the paper as discussing neither confidence intervals nor standard errors.

Paper 4

Classification: Excluded.

Comment: The paper is largely theoretical, and the small amount of empirical analysis it includes is entirely descriptive.

Paper 5

Classification: Excluded.

Comment: The paper is entirely theoretical.

Paper 6

Classification: Excluded.

Comment: The paper is entirely theoretical.

Paper 7

Classification: Discusses neither confidence intervals nor standard errors.

Comment: For many, though not all, of the paper's focal estimates, the confidence intervals are so narrow that the economic interpretation of any point within them would be virtually the same. See n. 1.

Paper 8

Classification: Discusses neither confidence intervals nor standard errors.

Paper 9

Classification: Excluded.

Comment: The paper is entirely theoretical.

Paper 10

Classification: Excluded.

Comment: The paper is entirely theoretical. 
Paper 11

Classification: Discusses neither confidence intervals nor standard errors.

Comment: Although the paper is largely theoretical, one section is devoted to an empirical application (pp. 442-447), with one table that reports standard errors for parameter estimates (p. 443). That section does not mention confidence intervals or standard errors in the text. The paper is marginal for inclusion in the sample.

Paper 12

Classification: Excluded.

Comment: The paper is an erratum.

\section{J PE, Issue 2}

Paper 1

Classification: Discusses standard errors in passing; does not discuss confidence intervals.

Comment: The only mention of a standard error occurs in the context of a discussion of the statistical significance of a result: "In our preferred specification shown in column 6, the net amplifier is $\$ 2.773$ billion. The standard error is $\$ 1,277$ [million], indicating that we can reject the null hypothesis that our estimate equals zero at the 1 percent level."

Paper 2

Classification: Discusses neither confidence intervals nor standard errors.

Paper 3

Classification: Discusses confidence intervals prominently.

Comment: The confidence interval for one of the paper's key estimates is reported in the conclusion. Since confidence intervals and standard errors are not discussed elsewhere, one could classify this paper as only discussing confidence intervals in passing.

Paper 4

Classification: Discusses neither confidence intervals nor standard errors.

Comment: In one footnote (n. 45, p. 614), the paper gives a standard error in reporting an estimate from a minor regression that is not presented in tables or figures.

Paper 5

Classification: Discusses neither confidence intervals nor standard errors.

Paper 6

Classification: Discusses neither confidence intervals nor standard errors.

Paper 7

Classification: Excluded.

Comment: The paper is entirely theoretical.

Paper 8

Classification: Excluded.

Comment: The paper is entirely theoretical.

Paper 9

Classification: Discusses neither confidence intervals nor standard errors.

Paper 10

Classification: Discusses standard errors in passing; does not discuss confidence intervals. 
Comment: Although the paper is largely theoretical, one section is devoted to estimating some parameters that enter into the model (pp. 874-878). That section makes one mention of standard errors in the text (p. 878). The paper is marginal for inclusion in the sample.

Paper 11

Classification: Discusses neither confidence intervals nor standard errors.

Paper 12

Classification: Excluded.

Comment: The paper is an erratum.

\section{J PE, Issue 3}

Paper 1

Classification: Excluded.

Comment: I am thanked for comments.

Paper 2

Classification: Discusses neither confidence intervals nor standard errors.

Paper 3

Classification: Discusses neither confidence intervals nor standard errors.

Comment: At one point, the paper compares the sizes of standard errors under different estimation methods, but it does not report any quantitative information about the magnitudes of the standard errors (p. 1087).

Paper 4

Classification: Excluded.

Comment: The paper is entirely theoretical.

Paper 5

Classification: Excluded.

Comment: The paper is entirely theoretical.

Paper 6

Classification: Discusses neither confidence intervals nor standard errors.

Paper 7

Classification: Discusses standard errors prominently; does not discuss confidence intervals.

Paper 8

Classification: Discusses standard errors in passing; does not discuss confidence intervals.

Comment: There is one mention of the point estimate and t-statistic for an estimate that is not particularly central to the paper's analysis (p. 1301). In addition, in a few places the paper mentions that the standard errors are sufficiently large that the equality of some coefficients cannot be rejected (pp. 1265 and 1290); but this is just another way of saying that the difference in the coefficients is not statistically significant, and does not convey any additional information about the standard errors.

Paper 9

Classification: Excluded.

Comment: The paper is largely theoretical, and the small amount of empirical analysis it includes is entirely descriptive. 
Paper 10

Classification: Discusses neither confidence intervals nor standard errors.

Paper 11

Classification: Discusses neither confidence intervals nor standard errors.

Comment: At one point, the paper reports standard errors from some minor regressions that are not reported in any tables or figures (p. 1433n).

Paper 12

Classification: Excluded.

Comment: I am thanked for comments.

\section{J PE, Issue 4}

Paper 1

Classification: Discusses neither confidence intervals nor standard errors.

Paper 2

Classification: Discusses neither confidence intervals nor standard errors.

Comment: At one point (p. 1512), the paper discusses the effects of different ways of computing standard errors; but the paper does not discuss the magnitude of the standard errors under any of these approaches. At another point (p. 1541), the paper refers to a minor estimate as "imprecisely estimated," but it does not elaborate and appears to be using "imprecisely estimated" as a synonym for "not statistically significant."

Paper 3

Classification: Discusses neither confidence intervals nor standard errors.

Comment: The paper contains a few passing references to parameters being precisely or imprecisely estimated, but nothing approaching a concrete discussion of the magnitudes of standard errors or the width of confidence intervals.

Paper 4

Classification: Discusses neither confidence intervals nor standard errors.

Comment: For many, though not all, of the paper's key estimates, the standard errors are very small and the confidence intervals quite narrow. Thus for these estimates, failing to discuss standard errors or confidence intervals fails to convey how extremely precise the estimates are. See n. 1.

Paper 5

Classification: Excluded.

Comment: This short paper's empirical application reports only qualitative information about previous studies. No standard errors or confidence intervals are reported in tables or figures.

Paper 6

Classification: Discusses confidence intervals prominently.

Comment: The paper does not report standard errors or confidence intervals for its main estimates in the text. However, it gauges the magnitude of focal effect by comparing its estimates with another effect, and repeatedly reports confidence intervals for the ratio of the two effects.

Paper 7

Classification: Discusses neither confidence intervals nor standard errors.

Comment: In two places (pp. 1713 and 1716), the paper mentions that the point estimates are 
comfortably within model-simulated confidence intervals. But there is no mention of any quantitative information about the confidence intervals in the text.

Paper 8

Classification: Excluded.

Comment: The paper is entirely theoretical.

Paper 9

Classification: Discusses neither confidence intervals nor standard errors.

Paper 10

Classification: Discusses confidence intervals in passing; does not discuss standard errors.

Comment: The text of the paper reports the confidence interval for one estimate that is reported in a table or figure (p. 1857). (In addition, standard errors for some minor estimates that are not reported in tables or figures are reported at several points in the text; see pp. 1851n, 1855, and 1856. The paper also discusses the direction in which aspects of the estimation move standard errors, but without providing information about the magnitude of the standard errors; see pp. 1841 and 1849.)

Paper 11

Classification: Discusses neither confidence intervals nor standard errors.

Paper 12

Classification: Excluded.

Comment: The paper is entirely theoretical, with the exception of a brief empirical example where no standard errors or confidence intervals are reported in tables or figures (pp. 10771078). The paper also reports the results of some regressions using simulated data, again without standard errors or confidence intervals (pp. 1952- 1954 and 1971- 1972).

\section{J PE, Issue 5}

Paper 1

Classification: Excluded.

Comment: The paper is entirely theoretical.

Paper 2

Classification: Excluded.

Comment: The paper is mainly theoretical, with a small amount of empirical work that is purely descriptive.

Paper 3

Classification: Discusses confidence intervals in passing; does not discuss standard errors.

Comment: At one point, in discussing two point estimates that are small, the paper mentions that one confidence interval is very narrow while other is moderate (p. 2136).

Paper 4

Classification: Discusses confidence intervals in passing; does not discuss standard errors.

Comment: At one point, the paper refers to an estimate as "a precisely estimated -0.014 " (p. 2154). Although this could be interpreted only as meaning that the estimate is highly statistically significant, it implies that the confidence interval is quite narrow (which it is).

Paper 5

Classification: Excluded. 
Comment: The paper is mainly theoretical, with a small amount of empirical work that is purely descriptive.

Paper 6

Classification: Discusses neither confidence intervals nor standard errors.

Comment: For many, though not all, of the paper's focal estimates, the confidence intervals are so narrow that the economic interpretation of any point within them would be virtually the same. See n. 1.

Paper 7

Classification: Discusses neither confidence intervals nor standard errors.

\section{Quarterly J ournal of Economics}

\section{QJ E, Issue 1}

\section{Paper 1}

Classification: Excluded.

Comment: The paper's data analysis is descriptive. Some of those descriptions can be thought of as parameter estimates (see Tables III and IV on pp. 31-33, for example). However, because the paper does not report standard errors or confidence intervals in any tables or figures, I classify the paper as descriptive. The paper is marginal for inclusion in the sample. If it were included, it would be classified as discussing neither confidence intervals nor standard errors.

Paper 2

Classification: Discusses neither confidence intervals nor standard errors.

Paper 3

Classification: Discusses neither confidence intervals nor standard errors.

Comment: In one footnote (n. 17, p. 132), the paper gives a standard error in reporting an estimate from a minor regression that is not presented in tables or figures.

Paper 4

Classification: Discusses neither confidence intervals nor standard errors.

Paper 5

Classification: Discusses confidence intervals prominently.

Comment: The paper's central result is a negative one. The paper discusses confidence intervals (particularly their upper end) and their economic interpretation for many of its main estimates in numerous places, including in the abstract.

Paper 6

Classification: Discusses neither confidence intervals nor standard errors.

Comment: The paper's reference to "at most a small effect" in the abstract and similar comments elsewhere refer to the upper bound of ranges of point estimates under different specifications, not to the upper bound of confidence intervals.

Paper 7

Classification: Discusses neither confidence intervals nor standard errors.

Comment: In one footnote (n. 20, p. 382), the paper gives standard errors in reporting estimates from some minor regressions that are not presented in tables or figures. 
Paper 8

Classification: Discusses confidence intervals prominently.

Comment: The paper reports standards errors for all of its main estimates in the text, including some in the introduction. It also reports confidence intervals for some of its main estimates at one point in the paper (p. 436). (Less importantly, the paper gives standard errors and confidence intervals in reporting some estimates from minor regressions that are not presented in tables or figures; see pp. 429-430.)

Paper 9

Classification: Excluded.

Comment: The paper's data analysis is descriptive. Some of those descriptions can be thought of as parameter estimates (see Figure IV on p. 480 and Table I on p. 486, for example). However, because the paper does not report standard errors or confidence intervals in its tables and figures, I classify the paper as descriptive. The paper is marginal for inclusion in the sample. If it were included, it would be classified as discussing neither confidence intervals nor standard errors.

Paper 10

Classification: Discusses neither confidence intervals nor standard errors.

Comment: The paper reports two t-statistics in the text (pp. 522 and 524), but they are not reported with the associated point estimates. Thus they provide no information about magnitudes.

\section{QJ E, Issue 2}

Paper 1

Classification: Excluded.

Comment: The paper's data analysis is descriptive. Some of those descriptions can be thought of as parameter estimates (see Tables V and VI on pp. 586 and 588, for example). However, because the paper does not report standard errors or confidence intervals in its tables, I classify the paper as descriptive. The paper is marginal for inclusion in the sample. If it were included, it would be classified as discussing neither confidence intervals nor standard errors.

Paper 2

Classification: Excluded.

Comment: The paper's data analysis is descriptive (for essentially the full population of interest).

Paper 3

Classification: Discusses standard errors in passing; does not discuss confidence intervals.

Comment: There is one mention of the standard error for an estimate that is not particularly central to the paper's analysis (p. 686).

Paper 4

Classification: Discusses standard errors prominently; does not discuss confidence intervals. Comment: Standard errors are reported in numerous places, including in the abstract.

Paper 5

Classification: Discusses neither confidence intervals nor standard errors.

Comment: The closest the paper comes to discussing standard errors or confidence intervals are a statement that in unreported results, "we are unable to identify with precision" effects on some components of an outcome variable (p. 818n); a discussion of various ways of computing the standard errors that does not include any quantitative information about the magnitude of the standard errors (p. 829); and a statement that "there are probably large standard errors" for some 
numbers reported in a table that does not include standard errors (p. 834).

Paper 6

Classification: Discusses confidence intervals prominently.

Comment: The paper reports confidence intervals for two of its focal estimates in the text (pp. 886 and 888). (Less importantly, it also reports standard errors at various points in the text for specifications, control variables, or ways of computing standard errors that are not reported in tables or figures; see pp. 872n, 876n, 878, 880, and 886n.)

Paper 7

Classification: Discusses neither confidence intervals nor standard errors.

Paper 8

Classification: Discusses neither confidence intervals nor standard errors.

Paper 9

Classification: Discusses neither confidence intervals nor standard errors.

Paper 10

Classification: Discusses standard errors in passing; does not discuss confidence intervals.

Comment: Although the paper has a large statistical component, there is only one table or figure that reports standard errors or confidence intervals (Table VII, p. 1114), and the text does not include any discussion of standard errors or confidence intervals associated with those estimates. However, various parameter estimates that are not reported in tables or figures, some of which are relatively important to the paper, are reported in the text together with the associated standard errors. It is not clear how this paper should be classified, or whether it should be included in the sample at all.

\section{QJ E, Issue 3}

Paper 1

Classification: Discusses neither confidence intervals nor standard errors.

Paper 2

Classification: Discusses neither confidence intervals nor standard errors.

Comment: In several places, standard errors are reported in the text for minor estimates that are not reported in any tables or figures in the paper (pp. 1186, 1199, and 1204n).

Paper 3

Classification: Excluded.

Comment: The paper's data is entirely descriptive. Some of those descriptions can be thought of as parameter estimates (see Figures VIII, IX, and XI, for example). However, because the paper does not report standard errors or confidence intervals in its figures, I classify the paper as descriptive. The paper is marginal for inclusion in the sample. If it were included, it would be classified as discussing neither confidence intervals nor standard errors

Paper 4

Classification: Discusses neither confidence intervals nor standard errors.

Paper 5

Classification: Discusses confidence intervals prominently. literature.

Comment: The paper uses confidence intervals to compare its findings with the prior 
Paper 6

Classification: Discusses confidence intervals prominently.

Paper 7

Classification: Discusses confidence intervals prominently.

Paper 8

Classification: Discusses neither confidence intervals nor standard errors.

Paper 9

Classification: Discusses neither confidence intervals nor standard errors.

Paper 10

Classification: Discusses neither confidence intervals nor standard errors.

\section{QJ E, Issue 4}

Paper 1

Classification: Discusses standard errors prominently; does not discuss confidence intervals.

Paper 2

Classification: Discusses confidence intervals prominently.

Comment: The paper uses confidence intervals to compare its findings with the prior literature.

Paper 3

Classification: Discusses confidence intervals prominently.

Paper 4

Classification: Discusses neither confidence intervals nor standard errors.

Comment: For many, but not all, of the paper's key estimates, the standard errors are very small and the confidence intervals quite narrow. Thus for these estimates, failing to discuss standard errors or confidence intervals fails to convey how extremely precise the estimates are. See n. 1.

Paper 5

Classification: Discusses neither confidence intervals nor standard errors.

Comment: Although most of the paper is theoretical or descriptive, one part (pp. 1930-1935, including Tables XI and XII) estimates parameters statistically and reports standard errors in the tables.

Paper 6

Classification: Discusses confidence intervals prominently.

Comment: Most of the paper is an econometric analysis of standard estimates of confidence intervals from a particular empirical approach. The paper's empirical applications focus on the relative sizes of confidence intervals using different approaches, not the economic interpretation of any values within the confidence intervals or of the bounds of the intervals. The one exception is that at one point, the paper alludes to the economic interpretation of a parameter value at the extreme of a confidence interval (pp. 2003-2004). The paper is marginal for inclusion in the sample.

Paper 7

Classification: Discusses standard errors prominently; does not discuss confidence intervals. 


\section{Paper 8}

Classification: Discusses neither confidence intervals nor standard errors.

\section{Paper 9}

Classification: Discusses standard errors prominently; does not discuss confidence intervals.

Paper 10

Classification: Discusses confidence intervals in passing; does not discuss standard errors.

Comment: The paper mentions the upper bounds of the confidence intervals for a few estimates that are not central to the paper (pp. 2225 and 2225n). 